\title{
The Gopala-Hemachandra universal code determined by straight lines
}

Joydeb Pal ${ }^{\mathrm{a}}$, Monojit Das ${ }^{\mathrm{b}}$

${ }^{a}$ Department of Mathematics, National Institute of Technology Durgapur, Burdwan, W.B., Pin-713209, India.

${ }^{b}$ Shibpur Dinobundhoo Institution (College), Howrah, W.B., Pin-711102, India.

\begin{abstract}
Variation on the Fibonacci universal code, known as Gopala-Hemachandra (or GH) code, is mainly used in data compression and cryptography as it is a self-synchronizing code. In 2010, Basu and Prasad showed that Gopala-Hemachandra code $\mathrm{GH}_{\mathrm{a}}(\mathrm{n})$ exists for $-20 \leqslant a \leqslant-2$ and $1 \leqslant n \leqslant 100$ as well as there are $m$ consecutive non-existing Gopala-Hemachandra codewords in $\mathrm{GH}_{-(4+\mathrm{m})}(\mathrm{n})$ column where $1 \leqslant \mathrm{~m} \leqslant 16$. In this paper, we have introduced GH code straight line in two-dimensional space where each integral point $(a, n)$ on the GH code straight line represents a unique GH codeword. GH code straight lines confirm the existence of GH codewords for any integer $n \geqslant 1$ and integer $a \leqslant-2$. Moreover, for a given parameter ( $a, n)$, we have introduced two methods to check whether GH codeword exists or not.
\end{abstract}

Keywords: Fibonacci numbers, Fibonacci coding, Gopala-Hemachandra sequence, Gopala-Hemachandra code, Zeckendorf's representation.

2010 MSC: 94B25, 11B39, $11 \mathrm{T71}$.

(C)2019 All rights reserved.

\section{Introduction}

The Fibonacci sequence is a sequence of positive integers whose terms are defined by the recurrence relation $F(n)=F(n-1)+F(n-2), \forall n>2$ with $F(1)=1$ and $F(2)=2$. The Fibonacci universal code encodes positive integers into binary codewords. The rule of encoding is performed by using the Zeckendorf's representation of positive integers. The Zeckendorf's theorem states that every positive integer has a unique representation as the sum of non-consecutive Fibonacci numbers [9]. Let, $n$ be a positive integer which can be written as a sum of Fibonacci numbers as $n=\sum_{k=1}^{l} a_{k} F(k)$ where $a_{k} \in\{0,1\}$ such that the string $a_{1} a_{2} a_{3} \cdots a_{l}$ does not contain any consecutive 1 's, where $F(k)$ is the $k^{\text {th }}$ Fibonacci number. For an example, 14 can be represented as $F(1)+F(2)+F(3)+F(5)$ or $F(1)+F(4)+F(5)$ or $F(1)+F(6)$ but the Zeckendorf's representation will be $F(1)+F(6)$. So, the Zeckendorf's representation gives a unique binary string say $a_{1} a_{2} a_{3} \cdots a_{l}$ for a positive integer $n$. Then $a_{1} a_{2} a_{3} \cdots a_{l} 1$ will be the Fibonacci representation of $n$. The codewords end with 11 and have no other consecutive 1's in between it. The Fibonacci

\footnotetext{
*Corresponding author

Email addresses: joydebpal77@gmail.com (Joydeb Pal), monojitbhu@gmail.com (Monojit Das)
}

doi: $10.22436 /$ jmcs.019.03.03

Received: 2018-11-23 Revised: 2019-02-22 Accepted: 2019-04-19 
universal code is a uniquely decodable binary code of variable size since it is a prefix code. The main disadvantage of this representation is that the size $n$ of the set of integers has to be known in advance since it determines the code size as $1+\left\lfloor\log _{2} n\right\rfloor$. Since Fibonacci codes do not have consecutive 1 bits, they restricts the number of binary patterns available for such codes and in comparison, they are longer than the other codes. But they perform better in contrast to the Elias code [3] until the number of source messages is not very large. The Fibonacci universal code has the additional attribute of robustness, which manifests itself by the local containment of errors.

Fibonacci universal code is much significant than other universal codes because it has the inherent property to recover data from a damaged stream easily. In channel coding, if a single bit is changed, the data comes after it will not be correctly identified. On the other hand, with the Fibonacci universal coding, a changed bit may cause one token to be read as two, or cause two tokens to be misread as one, but reading a 0 from the stream will stop the errors from propagating further. Since the only stream that has no 0 in it is a stream of 11 tokens, the total edit distance between a stream damaged by a single bit error and the original stream is at most three.

Around the year 1150, Hemachandra introduced the Gopala-Hemachandra (GH) sequence. It is worth noting that Gopala studied these numbers in about the year 1135. The GH sequences are correlated with the Fibonacci sequence have probable applications in cryptography and coding theory presented in several studies $[1,8]$.

In 2007, Thomas [8] proposed Variant Fibonacci sequence which was also called Gopala-Hemachandra $(\mathrm{GH})$ sequence denoted by $\mathrm{VF}_{\mathrm{a}}(\mathrm{n})$. He had shown that the Variant Fibonacci sequence cannot be generated for all values of $a$. He gave a table in which he showed that for $a=-5$ there is no Zeckendorf's representation for $n=5,12$. But he did not give a specific range for a.

In 2010, Basu and Prasad [1] invented an improvement of [8] in which they showed the existence of the Gopala-Hemachandra code $\mathrm{GH}_{\mathrm{a}}(\mathrm{n})$ for $-20 \leqslant \mathrm{a} \leqslant-2$, and $1 \leqslant n \leqslant 100$. Besides, they described that there are at most $m$ consecutive non-existing $\mathrm{GH}$ codewords in a column of $\mathrm{GH}_{-(4+\mathrm{m})}$ for $1 \leqslant \mathrm{~m} \leqslant 16$, which are given in Tables 1, 2, 3, and 4.

In this paper, we extend the availability of GH universal code. Here we define a GH code straight line to obtain a GH code. Thereafter, we propose two methods to check the existence of GH code for a given parameter $(a, n)$, where $a \leqslant-2$ and $n$ is any positive integer.

In this paper, we use the following two properties.

Property 1.1. In the Zeckendorf's representation of any two consecutive positive integers, it is not possible that both the coefficients of $\mathrm{F}(1)$ are 1 .

Property 1.2. In the Zeckendorf's representation of any two consecutive positive integers $\mathrm{n}$ and $\mathrm{n}+1$, if the coefficient of $\mathrm{F}(1)$ is 0 for both, then the coefficient of $\mathrm{F}(2)$ is 1 for $n$, and 0 for $n+1$.

In Section 2, we discuss Gopala-Hemachandra sequence and codes and establish a relation between Gopala-Hemachandra sequence and the Fibonacci sequence. In Section 3, we introduce GH code straight line and give some properties on the Gopala-Hemachandra universal code. Two methods with illustrations are given in Section 4, which provide the existence or non-existence of GH code corresponding to a parameter $(a, n)$. In the final section, we conclude the article.

\section{Gopala-Hemachandra sequence and codes}

The sequence $\{a, b, a+b, a+2 b, 2 a+3 b, \ldots\}$ for any $a, b \in \mathbb{Z}$, named as Gopala-Hemachandra $(\mathrm{GH})$ sequence is the more general variant Fibonacci sequence. For the pair of values $a=1$ and $b=2$, it represents the Fibonacci sequence. The historical details of these sequences are discussed in $[4,5,7]$.

The variant of $\mathrm{GH}$ coding scheme is obtained by second order variant $\mathrm{GH}$ sequence $V \mathrm{~F}_{\mathrm{a}}(\mathrm{k})$ such that $\mathrm{b}=1-\mathrm{a}, \mathrm{VF}_{\mathrm{a}}(1)=\mathrm{a}, \mathrm{VF}_{\mathrm{a}}(2)=1-\mathrm{a}$ and for $\mathrm{k} \geqslant 3$,

$$
\mathrm{VF}_{\mathrm{a}}(\mathrm{k})=\mathrm{VF}_{\mathrm{a}}(\mathrm{k}-1)+\mathrm{VF}_{\mathrm{a}}(\mathrm{k}-2) .
$$


In 1960, Daykin [2] proved that only the standard Fibonacci sequence $F(k)$ gives a unique Zeckendorf's representation for all positive integers.

Table 1: GH Code $(1 \leqslant n \leqslant 50,-2 \leqslant a \leqslant-11)$.

\begin{tabular}{|c|c|c|c|c|c|c|c|c|c|c|}
\hline$n$ & $\mathrm{GH}_{-2}$ & $\mathrm{GH}_{-3}$ & $\mathrm{GH}_{-4}$ & $\mathrm{GH}_{-5}$ & $\mathrm{GH}_{-6}$ & $\mathrm{GH}_{-7}$ & $\mathrm{GH}_{-8}$ & $\mathrm{GH}_{-9}$ & $\mathrm{GH}_{-10}$ & $\mathrm{GH}_{-11}$ \\
\hline 1 & 0011 & 0011 & 0011 & 0011 & 0011 & 0011 & 0011 & 0011 & 0011 & 0011 \\
\hline 2 & 10011 & 10011 & 10011 & 10011 & 10011 & 10011 & 10011 & 10011 & 10011 & 10011 \\
\hline 3 & 011 & 100011 & 100011 & 100011 & 100011 & 100011 & 100011 & 100011 & 100011 & 100011 \\
\hline 4 & 00011 & 011 & 101011 & 101011 & 101011 & 101011 & 101011 & 101011 & 101011 & 101011 \\
\hline 5 & 000011 & 00011 & 011 & N/A & N/A & N/A & N/A & N/A & N/A & N/A \\
\hline 6 & 001011 & 000011 & 00011 & 011 & N/A & $\mathrm{N} / \mathrm{A}$ & N/A & N/A & N/A & N/A \\
\hline 7 & 01011 & 001011 & 000011 & 00011 & 011 & N/A & N/A & N/A & N/A & N/A \\
\hline 8 & 1010011 & 1000011 & 001011 & 000011 & 00011 & 011 & N/A & N/A & N/A & N/A \\
\hline 9 & 0000011 & 1010011 & 1000011 & 001011 & 000011 & 00011 & 011 & N/A & N/A & N/A \\
\hline 10 & 0010011 & 010011 & 1010011 & 1000011 & 001011 & 000011 & 00011 & 011 & N/A & N/A \\
\hline 11 & 1001011 & 0000011 & 01011 & 1010011 & 1000011 & 001011 & 000011 & 00011 & 011 & N/A \\
\hline 12 & 0100011 & 0010011 & 010011 & N/A & 1010011 & 1000011 & 001011 & 000011 & 00011 & 011 \\
\hline 13 & 10100011 & 1001011 & 0000011 & 01011 & N/A & 1010011 & 1000011 & 001011 & 000011 & 00011 \\
\hline 14 & 00000011 & 10000011 & 0010011 & 010011 & N/A & N/A & 1010011 & 1000011 & 001011 & 000011 \\
\hline 15 & 00100011 & 10100011 & 1001011 & 0000011 & 01011 & $\mathrm{~N} / \mathrm{A}$ & N/A & 1010011 & 1000011 & 001011 \\
\hline 16 & 10010011 & 0001011 & 10000011 & 0010011 & 010011 & N/A & $\mathrm{N} / \mathrm{A}$ & N/A & 1010011 & 1000011 \\
\hline 17 & 01000011 & 00000011 & 10100011 & 1001011 & 0000011 & 01011 & N/A & N/A & N/A & 1010011 \\
\hline 18 & 10101011 & 00100011 & 0100011 & 10000011 & 0010011 & 010011 & N/A & N/A & $\mathrm{N} / \mathrm{A}$ & N/A \\
\hline 19 & 00001011 & 10010011 & 0001011 & 10100011 & 1001011 & 1000011 & 01011 & N/A & N/A & N/A \\
\hline 20 & 00101011 & 0101011 & 00000011 & N/A & 10000011 & 0010011 & 010011 & N/A & N/A & N/A \\
\hline 21 & 01010011 & 10101011 & 00100011 & 0100011 & 10100011 & 1001011 & 0000011 & 01011 & N/A & N/A \\
\hline 22 & 101000011 & 00010011 & 10010011 & 0001011 & N/A & 10000011 & 0010011 & 010011 & $\mathrm{~N} / \mathrm{A}$ & N/A \\
\hline 23 & 000000011 & 00001011 & 10001011 & 00000011 & N/A & 10100011 & 1001011 & 0000011 & 01011 & N/A \\
\hline 24 & 001000011 & 00101011 & 0101011 & 00100011 & 0100011 & N/A & 10000011 & 0010011 & 010011 & N/A \\
\hline 25 & 100100011 & 100000011 & 01000011 & 10010011 & 0001011 & N/A & 10100011 & 1001011 & 0000011 & 01011 \\
\hline 26 & 100010011 & 01010011 & 00010011 & 10001011 & 00000011 & N/A & N/A & 10000011 & 0010011 & 010011 \\
\hline 27 & 101010011 & 01001011 & 00001011 & 10101011 & 00100011 & 0100011 & N/A & 10100011 & 1001011 & 0000011 \\
\hline 28 & 000010011 & 000000011 & 00101011 & 0101011 & 10010011 & 0001011 & N/A & N/A & 10000011 & 0010011 \\
\hline 29 & 001010011 & 001000011 & 100000011 & 01000011 & 10001011 & 00000011 & N/A & N/A & 10100011 & 1001011 \\
\hline 30 & 010100011 & 100100011 & 101000011 & 00010011 & 10101011 & 00100011 & 0100011 & N/A & N/A & 10000011 \\
\hline 31 & 101001011 & 100010011 & 01010011 & 00001011 & N/A & 10010011 & 0001011 & N/A & N/A & 10100011 \\
\hline 32 & 000001011 & 101010011 & 01001011 & 00101011 & 0101011 & 10001011 & 00000011 & N/A & $\mathrm{N} / \mathrm{A}$ & N/A \\
\hline 33 & 001001011 & 000100011 & 000000011 & 100000011 & 01000011 & 10101011 & 00100011 & 0100011 & $\mathrm{~N} / \mathrm{A}$ & N/A \\
\hline 34 & 100101011 & 000010011 & 001000011 & 101000011 & 00010011 & N/A & 10010011 & 0001011 & $\mathrm{~N} / \mathrm{A}$ & N/A \\
\hline 35 & 010001011 & 001010011 & 100100011 & N/A & 00001011 & N/A & 10001011 & 00000011 & N/A & N/A \\
\hline 36 & 1010000011 & 100001011 & 100010011 & 01010011 & 00101011 & 0101011 & 10101011 & 00100011 & 0100011 & N/A \\
\hline 37 & 0000000011 & 101001011 & 101010011 & 01001011 & 100000011 & 01000011 & N/A & 10010011 & 0001011 & N/A \\
\hline 38 & 0010000011 & 010010011 & 010000011 & 000000011 & 101000011 & 00010011 & N/A & 10001011 & 00000011 & N/A \\
\hline 39 & 1001000011 & 000001011 & 000100011 & 001000011 & N/A & 00001011 & N/A & 10101011 & 00100011 & 0100011 \\
\hline 40 & 1000100011 & 001001011 & 000010011 & 100100011 & N/A & 00101011 & 0101011 & N/A & 10010011 & 0001011 \\
\hline 41 & 1010100011 & 100101011 & 001010011 & 100010011 & 01010011 & 100000011 & 01000011 & N/A & 10001011 & 00000011 \\
\hline 42 & 0000100011 & 1000000011 & 100001011 & 101010011 & 01001011 & 101000011 & 00010011 & N/A & 10101011 & 00100011 \\
\hline 43 & 0010100011 & 010001011 & 101001011 & N/A & 000000011 & N/A & 00001011 & N/A & N/A & 10010011 \\
\hline 44 & 0101000011 & 000101011 & 010100011 & 010000011 & 001000011 & $\mathrm{~N} / \mathrm{A}$ & 00101011 & 0101011 & $\mathrm{~N} / \mathrm{A}$ & 10001011 \\
\hline 45 & 1010010011 & 0000000011 & 010010011 & 000100011 & 100100011 & $\mathrm{~N} / \mathrm{A}$ & 100000011 & 01000011 & N/A & 10101011 \\
\hline 46 & 0000010011 & 0010000011 & 000001011 & 000010011 & 100010011 & 01010011 & 101000011 & 00010011 & N/A & N/A \\
\hline 47 & 0010010011 & 1001000011 & 001001011 & 001010011 & 101010011 & 01001011 & N/A & 00001011 & N/A & N/A \\
\hline 48 & 1001010011 & 010101011 & 100101011 & 100001011 & N/A & 000000011 & N/A & 00101011 & 0101011 & N/A \\
\hline 49 & 1000001011 & 1010100011 & 1000000011 & 101001011 & N/A & 001000011 & N/A & 100000011 & 01000011 & N/A \\
\hline 50 & 1010001011 & 0001000011 & 1010000011 & N/A & 010000011 & 100100011 & N/A & 101000011 & 00010011 & N/A \\
\hline
\end{tabular}

Theorem 2.1. Let $\mathrm{F}(\mathrm{k})$ be the Fibonacci sequence with $\mathrm{F}(0)=\mathrm{F}(1)=1$. Then for $\mathrm{k} \geqslant 1$,

$$
\mathrm{VF}_{\mathrm{a}}(\mathrm{k})=\mathrm{F}(\mathrm{k}-2)-\mathrm{aF}(\mathrm{k}-4) \text {. }
$$

Proof. Consider, the GH sequence $\mathrm{VF}_{\mathrm{a}}(\mathrm{k})$ as $\{a, 1-a, 1,2-a, 3-a, 5-2 a, 8-3 a, 13-5 a, 21-8 a, \ldots\}$. Therefore, we have $\mathrm{VF}_{\mathrm{a}}(1)=\mathrm{a}=0-\mathrm{a}(-1)=\mathrm{F}(-1)-\mathrm{aF}(-3)=\mathrm{F}(1-2)-\mathrm{aF}(1-4)$ and $\mathrm{VF}_{\mathrm{a}}(2)=$ $1-\mathrm{a}(1)=\mathrm{F}(0)-\mathrm{aF}(-2)=\mathrm{F}(2-2)-\mathrm{aF}(2-4)$, according to [6]. Thus the result is true for $k=1$ and 2 .

Let the result is true for $k=1,2,3, \ldots, m$. Then, $V F_{a}(m-1)=F(m-3)-a F(m-5)$ and $V F_{a}(m)=$ $F(m-2)-a F(m-4)$. Therefore, $V F_{a}(m+1)=V F_{a}(m)+V F_{a}(m-1)=F(m-3)-a F(m-5)+F(m-$ 2) $-\mathrm{aF}(\mathrm{m}-4)=(\mathrm{F}(\mathrm{m}-3)+\mathrm{F}(\mathrm{m}-2))-\mathrm{a}(\mathrm{F}(\mathrm{m}-5)+\mathrm{F}(\mathrm{m}-4))=\mathrm{F}(\mathrm{m}-1)-\mathrm{aF}(\mathrm{m}-3)=\mathrm{F}(\overline{m+1}-2)-$ $\mathrm{aF}(\overline{\mathrm{m}+1}-4)$.

Hence by the second principle of mathematical induction, we have

$$
\mathrm{VF}_{\mathrm{a}}(\mathrm{k})=\mathrm{F}(\mathrm{k}-2)-\mathrm{aF}(\mathrm{k}-4), \mathrm{k} \geqslant 1 .
$$

\section{Some properties on GH universal code for $a \leq-2$}

The GH codewords for $n=1,2,3,4$ always exist and are 0011, 10011, 100011, 101011 respectively for any $a \leqslant-2$ since binary representation of $1,2,3$, and 4 are independent of $a$. 
Table 2: GH Code $(51 \leqslant n \leqslant 100,-2 \leqslant a \leqslant-11)$.

\begin{tabular}{|c|c|c|c|c|c|c|c|c|c|c|}
\hline$n$ & $\mathrm{GH}_{-2}$ & $\mathrm{GH}_{-3}$ & $\mathrm{GH}_{-4}$ & $\mathrm{GH}_{-5}$ & $\mathrm{GH}_{-6}$ & $\mathrm{GH}_{-7}$ & $\mathrm{GH}_{-8}$ & $\mathrm{GH}_{-9}$ & $\mathrm{GH}_{-10}$ & $\mathrm{GH}_{-11}$ \\
\hline 51 & 0000001011 & 0000100011 & 010001011 & 010100011 & 000100011 & 100010011 & 01010011 & N/A & 00001011 & $\mathrm{~N} / \mathrm{A}$ \\
\hline 52 & 0010001011 & 0010100011 & 000101011 & 010010011 & 000010011 & 101010011 & 01001011 & N/A & 00101011 & 0101011 \\
\hline 53 & 0101010011 & 1000010011 & 0000000011 & 000001011 & 001010011 & N/A & 000000011 & N/A & 100000011 & 01000011 \\
\hline 54 & 0100001011 & 1010010011 & 0010000011 & 001001011 & 100001011 & N/A & 001000011 & N/A & 101000011 & 00010011 \\
\hline 55 & 0001001011 & 0100100011 & 1001000011 & 100101011 & 101001011 & N/A & 100100011 & N/A & N/A & 00001011 \\
\hline 56 & 0000101011 & 0000010011 & 1000100011 & 1000000011 & N/A & 010000011 & 100010011 & 01010011 & N/A & 00101011 \\
\hline 57 & 0010101011 & 0010010011 & 010101011 & 1010000011 & N/A & 000100011 & 101010011 & 01001011 & N/A & 100000011 \\
\hline 58 & 10000000011 & 1001010011 & 0100000011 & N/A & 010100011 & 000010011 & N/A & 000000011 & N/A & 101000011 \\
\hline 59 & 0100101011 & 1000001011 & 0001000011 & 010001011 & 010010011 & 001010011 & N/A & 001000011 & N/A & N/A \\
\hline 60 & 00000000011 & 0100010011 & 0000100011 & 000101011 & 000001011 & 100001011 & N/A & 100100011 & N/A & N/A \\
\hline 61 & 00100000011 & 0001010011 & 0010100011 & 0000000011 & 001001011 & 101001011 & $\mathrm{~N} / \mathrm{A}$ & 100010011 & 01010011 & N/A \\
\hline 62 & 10010000011 & 0000001011 & 1000010011 & 0010000011 & 100101011 & N/A & 010000011 & 101010011 & 01001011 & N/A \\
\hline 63 & 01000000011 & 0010001011 & 1010010011 & 1001000011 & 1000000011 & $\mathrm{~N} / \mathrm{A}$ & 000100011 & $\mathrm{~N} / \mathrm{A}$ & 000000011 & N/A \\
\hline 64 & 10101000011 & 1001001011 & 0101000011 & 1000100011 & 1010000011 & N/A & 000010011 & N/A & 001000011 & N/A \\
\hline 65 & 00001000011 & 1000101011 & 0100100011 & 1010100011 & N/A & 010100011 & 001010011 & N/A & 100100011 & N/A \\
\hline 66 & 00101000011 & 1010101011 & 0000010011 & 010101011 & N/A & 010010011 & 100001011 & N/A & 100010011 & 01010011 \\
\hline 67 & 10000100011 & 0001001011 & 0010010011 & 0100000011 & 010001011 & 000001011 & 101001011 & N/A & 101010011 & 01001011 \\
\hline 68 & 10100100011 & 0000101011 & 1001010011 & 0001000011 & 000101011 & 001001011 & N/A & 010000011 & N/A & 000000011 \\
\hline 69 & 00000100011 & 0010101011 & 1000001011 & 0000100011 & 0000000011 & 100101011 & N/A & 000100011 & N/A & 001000011 \\
\hline 70 & 00100100011 & 10000000011 & 1010001011 & 0010100011 & 0010000011 & 1000000011 & N/A & 000010011 & N/A & 100100011 \\
\hline 71 & 10010100011 & 0101001011 & 0100010011 & 1000010011 & 1001000011 & 1010000011 & N/A & 001010011 & N/A & 100010011 \\
\hline 72 & 01000100011 & 0100101011 & 0001010011 & 1010010011 & 1000100011 & N/A & 010100011 & 100001011 & N/A & 101010011 \\
\hline 73 & 10100010011 & 00000000011 & 0000001011 & N/A & 1010100011 & N/A & 010010011 & 101001011 & N/A & N/A \\
\hline 74 & 00000010011 & 00100000011 & 0010001011 & 0101000011 & N/A & N/A & 000001011 & N/A & 010000011 & N/A \\
\hline 75 & 00100010011 & 10010000011 & 1001001011 & 0100100011 & 010101011 & 010001011 & 001001011 & N/A & 000100011 & N/A \\
\hline 76 & 10010010011 & 10001000011 & 1000101011 & 0000010011 & 0100000011 & 000101011 & 100101011 & N/A & 000010011 & N/A \\
\hline 77 & 01000010011 & 10101000011 & 0101010011 & 0010010011 & 0001000011 & 0000000011 & 1000000011 & N/A & 001010011 & N/A \\
\hline 78 & 10101010011 & 00010000011 & 0100001011 & 1001010011 & 0000100011 & 0010000011 & 1010000011 & N/A & 100001011 & N/A \\
\hline 79 & 00001010011 & 00001000011 & 0001001011 & 1000001011 & 0010100011 & 1001000011 & N/A & 010100011 & 101001011 & N/A \\
\hline 80 & 00101010011 & 00101000011 & 0000101011 & 1010001011 & 1000010011 & 1000100011 & N/A & 010010011 & N/A & 010000011 \\
\hline 81 & 01010010011 & 10000100011 & 0010101011 & N/A & 1010010011 & 1010100011 & N/A & 000001011 & N/A & 000100011 \\
\hline 82 & 01001010011 & 10100100011 & 10000000011 & 0100010011 & $\mathrm{~N} / \mathrm{A}$ & N/A & N/A & 001001011 & N/A & 000010011 \\
\hline 83 & 00000001011 & 001000011 & 10100000011 & 0001010011 & N/A & $\mathrm{N} / \mathrm{A}$ & 010001011 & 100101011 & N/A & 001010011 \\
\hline 84 & 00100001011 & 00000100011 & 0101001011 & 0000001011 & 0101000011 & 010101011 & 000101011 & 1000000011 & N/A & 100001011 \\
\hline 85 & 10010001011 & 00100100011 & 0100101011 & 0010001011 & 0100100011 & 0100000011 & 0000000011 & 1010000011 & $\mathrm{~N} / \mathrm{A}$ & 101001011 \\
\hline 86 & 10001001011 & 10010100011 & 00000000011 & 1001001011 & 0000010011 & 0001000011 & 0010000011 & N/A & 010100011 & N/A \\
\hline 87 & 10101001011 & 10000010011 & 00000011 & 1000101011 & 0010010011 & 0000100011 & 1001000011 & N/A & 010010011 & N/A \\
\hline 88 & 00001001011 & 10100010011 & 010000011 & 1010101011 & 1001010011 & 0010100011 & 1000100011 & N/A & 000001011 & N/A \\
\hline 89 & 00101001011 & 00010100011 & 10001000011 & 0101010011 & 1000001011 & 1000010011 & 1010100011 & N/A & 001001011 & N/A \\
\hline 90 & 01010001011 & 00000010011 & 10101000011 & 0100001011 & 1010001011 & 1010010011 & N/A & N/A & 100101011 & N/A \\
\hline 91 & 10100101011 & 00100010011 & 01000000011 & 0001001011 & N/A & N/A & N/A & 010001011 & 1000000011 & N/A \\
\hline 92 & 00000101011 & 10010010011 & 00010000011 & 0000101011 & N/A & N/A & N/A & 000101011 & 1010000011 & N/A \\
\hline 93 & 00100101011 & 01010100011 & 00001000011 & 0010101011 & 0100010011 & N/A & 010101011 & 0000000011 & N/A & 010100011 \\
\hline 94 & 10010101011 & 10101010011 & 00101000011 & 10000000011 & 0001010011 & 0101000011 & 0100000011 & 0010000011 & N/A & 010010011 \\
\hline 95 & 01000101011 & 00010010011 & 10000100011 & 10100000011 & 0000001011 & 0100100011 & 0001000011 & 1001000011 & N/A & 000001011 \\
\hline 96 & 00010101011 & 00001010011 & 10100100011 & N/A & 0010001011 & 0000010011 & 0000100011 & 1000100011 & N/A & 001001011 \\
\hline 97 & 000000000011 & 00101010011 & 01010000011 & 0101001011 & 1001001011 & 0010010011 & 0010100011 & 1010100011 & N/A & 100101011 \\
\hline 98 & 001000000011 & 10000001011 & 01001000011 & 0100101011 & 1000101011 & 1001010011 & 1000010011 & N/A & N/A & 1000000011 \\
\hline 99 & 01010101011 & 01010010011 & 00000100011 & 00000000011 & 1010101011 & 1000001011 & 1010010011 & N/A & 010001011 & 1010000011 \\
\hline 100 & 100010000011 & 01001010011 & 00100100011 & 00100000011 & N/A & 1010001011 & N/A & N/A & 000101011 & N/A \\
\hline
\end{tabular}

Throughout this paper, we take $a, n$ and $j$ as integers. Now, we discuss the following properties of the GH code by considering $(a, n)$ as a point in $x y$-plane and with the help of Tables 1, 2, 3, and 4 .

Proposition 3.1. For $a \leqslant-2$, there are four straight lines $y+0 x=0+j$ for $j=1,2,3,4$ such that the four points $(a, 1-0 a),(a, 2-0 a),(a, 3-0 a)$, and $(a, 4-0 a)$ lie on these lines for $j=1,2,3,4$, respectively which give the respective GH codewords 0011, 10011, 100011, and 101011.

Proposition 3.2. For $a \leqslant-2$, there are six straight lines $y+x=0+j$ for $j=1,2, \ldots, 6$ such that the six points $(a, 1-a),(a, 2-a), \ldots,(a, 6-a)$ lie on these lines for $j=1,2, \ldots, 6$, respectively which give the respective GH codewords 011, 00011, 000011, 001011, 1000011, and 1010011.

Proposition 3.3. For $a \leqslant-2$, there are seven straight lines $y+2 x=2+j$ for $j=1,2, \ldots, 7$ such that the seven points $(a, 3-2 a),(a, 4-2 a), \ldots,(a, 9-2 a)$ lie on these lines for $j=1,2, \ldots, 7$, respectively which give the respective GH codewords 01011, 010011, 0000011, 0010011, 1001011, 10000011, and 10100011.

Proposition 3.4. For $a \leqslant-2$, there are seven straight lines $y+3 x=5+j$ for $j=1,2, \ldots, 7$ such that the seven points $(a, 6-3 a),(a, 7-3 a), \ldots,(a, 12-3 a)$ lie on these lines for $j=1,2, \ldots, 7$, respectively which give the respective GH codewords 0100011, 0001011, 00000011, 00100011, 10010011, 10001011, and 10101011.

Proposition 3.5. For $a \leqslant-2$, there are seven straight lines $y+4 x=7+j$ for $j=1,2, \ldots, 7$ such that the seven points $(a, 8-4 a),(a, 9-4 a), \ldots,(a, 14-4 a)$ lie on these lines for $j=1,2, \ldots, 7$, respectively which give the respective GH codewords 0101011, 01000011, 00010011, 00001011, 00101011, 100000011, and 101000011. 
Table 3: GH Code $(1 \leqslant n \leqslant 50,-12 \leqslant a \leqslant-20)$.

\begin{tabular}{|c|c|c|c|c|c|c|c|c|c|}
\hline $\mathrm{n}$ & $\mathrm{GH}_{-12}$ & $\mathrm{GH}_{-13}$ & $\mathrm{GH}_{-14}$ & $\mathrm{GH}_{-15}$ & $\mathrm{GH}_{-16}$ & $\mathrm{GH}_{-17}$ & $\mathrm{GH}_{-18}$ & $\mathrm{GH}_{-19}$ & $\mathrm{GH}_{-20}$ \\
\hline 1 & 0011 & 0011 & 0011 & 0011 & 0011 & 0011 & 0011 & 0011 & 0011 \\
\hline 2 & 10011 & 10011 & 10011 & 10011 & 10011 & 10011 & 10011 & 10011 & 10011 \\
\hline 3 & 100011 & 100011 & 100011 & 100011 & 100011 & 100011 & 100011 & 100011 & 100011 \\
\hline 4 & 101011 & 101011 & 101011 & 101011 & 101011 & 101011 & 101011 & 101011 & 101011 \\
\hline 5 & N/A & N/A & N/A & N/A & N/A & N/A & N/A & N/A & N/A \\
\hline 6 & N/A & N/A & N/A & N/A & N/A & N/A & N/A & N/A & N/A \\
\hline 7 & N/A & N/A & N/A & N/A & N/A & N/A & N/A & N/A & N/A \\
\hline 8 & N/A & N/A & N/A & N/A & N/A & N/A & N/A & N/A & N/A \\
\hline 9 & N/A & N/A & N/A & N/A & N/A & N/A & N/A & N/A & N/A \\
\hline 10 & N/A & N/A & N/A & N/A & N/A & N/A & N/A & N/A & N/A \\
\hline 11 & N/A & N/A & N/A & N/A & N/A & N/A & N/A & N/A & N/A \\
\hline 12 & $\mathrm{~N} / \mathrm{A}$ & N/A & N/A & N/A & N/A & N/A & $\mathrm{N} / \mathrm{A}$ & N/A & N/A \\
\hline 13 & 011 & N/A & N/A & N/A & N/A & N/A & N/A & N/A & N/A \\
\hline 14 & 00011 & 011 & N/A & N/A & N/A & N/A & N/A & N/A & N/A \\
\hline 15 & 000011 & 00011 & 011 & N/A & N/A & N/A & N/A & N/A & N/A \\
\hline 16 & 001011 & 000011 & 00011 & 011 & N/A & N/A & N/A & N/A & $\mathrm{N} / \mathrm{A}$ \\
\hline 17 & 1000011 & 001011 & 000011 & 00011 & 011 & N/A & $\mathrm{N} / \mathrm{A}$ & N/A & $\mathrm{N} / \mathrm{A}$ \\
\hline 18 & 1010011 & 1000011 & 001011 & 000011 & 00011 & 011 & N/A & N/A & N/A \\
\hline 19 & N/A & 1010011 & 1000011 & 001011 & 000011 & 00011 & 011 & N/A & N/A \\
\hline 20 & N/A & N/A & 1010011 & 1000011 & 001011 & 000011 & 00011 & 011 & N/A \\
\hline 21 & N/A & N/A & N/A & 1010011 & 1000011 & 001011 & 000011 & 00011 & 011 \\
\hline 22 & N/A & N/A & N/A & N/A & 1010011 & 1000011 & 001011 & 000011 & 00011 \\
\hline 23 & N/A & N/A & N/A & N/A & $\mathrm{N} / \mathrm{A}$ & 1010011 & 1000011 & 001011 & 000011 \\
\hline 24 & N/A & N/A & N/A & N/A & N/A & $\mathrm{N} / \mathrm{A}$ & 1010011 & 1000011 & 001011 \\
\hline 25 & $\mathrm{~N} / \mathrm{A}$ & $\mathrm{N} / \mathrm{A}$ & N/A & N/A & N/A & N/A & N/A & 1010011 & 1000011 \\
\hline 26 & N/A & N/A & N/A & N/A & N/A & N/A & N/A & N/A & 1010011 \\
\hline 27 & 01011 & N/A & N/A & N/A & N/A & N/A & $\mathrm{N} / \mathrm{A}$ & N/A & N/A \\
\hline 28 & 010011 & N/A & N/A & N/A & N/A & N/A & $\mathrm{N} / \mathrm{A}$ & N/A & N/A \\
\hline 29 & 0000011 & 01011 & $\mathrm{~N} / \mathrm{A}$ & N/A & N/A & N/A & N/A & N/A & N/A \\
\hline 30 & 0010011 & 010011 & N/A & N/A & N/A & N/A & N/A & N/A & N/A \\
\hline 31 & 1001011 & 0000011 & 01011 & N/A & N/A & N/A & N/A & N/A & N/A \\
\hline 32 & 10000011 & 0010011 & 010011 & N/A & N/A & N/A & N/A & N/A & N/A \\
\hline 33 & 10100011 & 1001011 & 0000011 & 01011 & N/A & N/A & N/A & N/A & N/A \\
\hline 34 & N/A & 10000011 & 0010011 & 010011 & N/A & $\mathrm{N} / \mathrm{A}$ & $\mathrm{N} / \mathrm{A}$ & N/A & N/A \\
\hline 35 & N/A & 10100011 & 1001011 & 0000011 & 01011 & N/A & N/A & N/A & $\mathrm{N} / \mathrm{A}$ \\
\hline 36 & N/A & N/A & 10000011 & 0010011 & 010011 & N/A & N/A & N/A & N/A \\
\hline 37 & N/A & N/A & 10100011 & 1001011 & 0000011 & 01011 & $\mathrm{~N} / \mathrm{A}$ & N/A & N/A \\
\hline 38 & N/A & N/A & N/A & 10000011 & 0010011 & 010011 & N/A & N/A & N/A \\
\hline 39 & N/A & N/A & $\mathrm{N} / \mathrm{A}$ & 10100011 & 1001011 & 0000011 & 01011 & N/A & N/A \\
\hline 40 & N/A & N/A & N/A & $\mathrm{N} / \mathrm{A}$ & 10000011 & 0010011 & 010011 & N/A & N/A \\
\hline 41 & N/A & N/A & N/A & N/A & 10100011 & 1001011 & 0000011 & 01011 & N/A \\
\hline 42 & 0100011 & N/A & N/A & N/A & N/A & 10000011 & 0010011 & 010011 & $\mathrm{~N} / \mathrm{A}$ \\
\hline 43 & 0001011 & N/A & N/A & N/A & N/A & 10100011 & 1001011 & 0000011 & 01011 \\
\hline 44 & 00000011 & N/A & N/A & N/A & N/A & N/A & 10000011 & 0010011 & 010011 \\
\hline 45 & 00100011 & 0100011 & N/A & N/A & N/A & N/A & 10100011 & 1001011 & 0000011 \\
\hline 46 & 10010011 & 0001011 & N/A & N/A & N/A & N/A & N/A & 10000011 & 0010011 \\
\hline 47 & 10001011 & 00000011 & N/A & N/A & N/A & N/A & N/A & 10100011 & 1001011 \\
\hline 48 & 10101011 & 00100011 & 0100011 & N/A & N/A & $\mathrm{N} / \mathrm{A}$ & N/A & N/A & 10000011 \\
\hline 49 & N/A & 10010011 & 0001011 & N/A & N/A & $\mathrm{N} / \mathrm{A}$ & $\mathrm{N} / \mathrm{A}$ & N/A & 10100011 \\
\hline 50 & N/A & 10001011 & 00000011 & N/A & N/A & N/A & N/A & N/A & N/A \\
\hline
\end{tabular}

Proposition 3.6. For $a \leqslant-2$, there are seven straight lines $y+5 x=10+j$ for $j=1,2, \ldots, 7$ such that the seven points $(a, 11-5 a),(a, 12-5 a), \ldots,(a, 17-5 a)$ lie on these lines for $j=1,2, \ldots, 7$, respectively which give the respective GH codewords 01010011, 01001011, 000000011, 001000011, 100100011, 100010011, and 101010011.

Proposition 3.7. For $a \leqslant-2$, there are six straight lines $y+6 x=13+j$ for $j=1,2, \ldots, 6$ such that the six points $(a, 14-6 a),(a, 15-6 a), \ldots,(a, 19-6 a)$ lie on these lines for $j=1,2, \ldots, 6$, respectively which give the respective GH codewords 010000011, 000100011, 000010011, 001010011, 100001011, and 101001011.

Proposition 3.8. For $a \leqslant-2$, there are seven straight lines $y+7 x=15+j$ for $j=1,2, \ldots, 7$ such that the seven points $(a, 16-7 a),(a, 17-7 a), \ldots,(a, 22-7 a)$ lie on these lines for $j=1,2, \ldots, 7$, respectively which give the respective GH codewords 010100011, 010010011, 000001011, 001001011, 100101011, 1000000011, and 1010000011.

Proposition 3.9. For $a \leqslant-2$, there are seven straight lines $y+8 x=18+j$ for $j=1,2, \ldots, 7$ such that the seven points $(a, 19-8 a),(a, 20-8 a), \ldots,(a, 25-8 a)$ lie on these lines for $j=1,2, \ldots, 7$, respectively which give the respective GH codewords 010001011, 000101011, 0000000011, 0010000011, 1001000011, 1000100011, and 1010100011.

Proposition 3.10. For $a \leqslant-2$, there are seven straight lines $y+9 x=20+j$ for $j=1,2, \ldots, 7$ such that the seven points $(a, 21-9 a),(a, 22-9 a), \ldots,(a, 27-9 a)$ lie on these lines for $j=1,2, \ldots, 7$, respectively which give the respective GH codewords 010101011, 0100000011, 0001000011, 0000100011, 0010100011, 1000010011, and 1010010011. 
Table 4: GH Code $(51 \leqslant n \leqslant 100,-12 \leqslant a \leqslant-20)$.

\begin{tabular}{|c|c|c|c|c|c|c|c|c|c|}
\hline$n$ & $\mathrm{GH}_{-12}$ & $\mathrm{GH}_{-13}$ & $\mathrm{GH}_{-14}$ & $\mathrm{GH}_{-15}$ & $\mathrm{GH}_{-16}$ & $\mathrm{GH}_{-17}$ & $\mathrm{GH}_{-18}$ & $\mathrm{GH}_{-19}$ & $\mathrm{GH}_{-20}$ \\
\hline 51 & N/A & 10101011 & 00100011 & 0100011 & N/A & N/A & N/A & N/A & N/A \\
\hline 52 & N/A & N/A & 10010011 & 0001011 & N/A & N/A & N/A & N/A & N/A \\
\hline 53 & N/A & $\mathrm{N} / \mathrm{A}$ & 10001011 & 00000011 & N/A & N/A & N/A & N/A & N/A \\
\hline 54 & N/A & $\mathrm{N} / \mathrm{A}$ & 10101011 & 00100011 & 0100011 & N/A & N/A & N/A & N/A \\
\hline 55 & N/A & $\mathrm{N} / \mathrm{A}$ & N/A & 10010011 & 0001011 & N/A & N/A & $\mathrm{N} / \mathrm{A}$ & N/A \\
\hline 56 & 0101011 & $\mathrm{~N} / \mathrm{A}$ & N/A & 10001011 & 00000011 & N/A & N/A & N/A & N/A \\
\hline 57 & 01000011 & N/A & N/A & 10101011 & 00100011 & 0100011 & N/A & N/A & N/A \\
\hline 58 & 00010011 & $\mathrm{~N} / \mathrm{A}$ & N/A & N/A & 10010011 & 0001011 & N/A & $\mathrm{N} / \mathrm{A}$ & N/A \\
\hline 59 & 00001011 & N/A & N/A & N/A & 10001011 & 00000011 & N/A & $\mathrm{N} / \mathrm{A}$ & N/A \\
\hline 60 & 00101011 & 0101011 & N/A & N/A & 10101011 & 00100011 & 0100011 & N/A & N/A \\
\hline 61 & 100000011 & 01000011 & N/A & N/A & N/A & 10010011 & 0001011 & N/A & N/A \\
\hline 62 & 101000011 & 00010011 & N/A & N/A & N/A & 10001011 & 00000011 & N/A & N/A \\
\hline 63 & N/A & 00001011 & N/A & N/A & $\mathrm{N} / \mathrm{A}$ & 10101011 & 00100011 & 0100011 & N/A \\
\hline 64 & $\mathrm{~N} / \mathrm{A}$ & 00101011 & 0101011 & N/A & $\mathrm{N} / \mathrm{A}$ & N/A & 10010011 & 0001011 & N/A \\
\hline 65 & N/A & 100000011 & 01000011 & N/A & N/A & $\mathrm{N} / \mathrm{A}$ & 10001011 & 00000011 & N/A \\
\hline 66 & $\mathrm{~N} / \mathrm{A}$ & 101000011 & 00010011 & N/A & N/A & N/A & 10101011 & 00100011 & 0100011 \\
\hline 67 & $\mathrm{~N} / \mathrm{A}$ & N/A & 00001011 & N/A & N/A & N/A & N/A & 10010011 & 0001011 \\
\hline 68 & $\mathrm{~N} / \mathrm{A}$ & N/A & 00101011 & 0101011 & N/A & N/A & N/A & 10001011 & 00000011 \\
\hline 69 & $\mathrm{~N} / \mathrm{A}$ & $\mathrm{N} / \mathrm{A}$ & 100000011 & 01000011 & $\mathrm{~N} / \mathrm{A}$ & N/A & $\mathrm{N} / \mathrm{A}$ & 10101011 & 00100011 \\
\hline 70 & N/A & $\mathrm{N} / \mathrm{A}$ & 101000011 & 00010011 & $\mathrm{~N} / \mathrm{A}$ & N/A & N/A & N/A & 10010011 \\
\hline 71 & 01010011 & N/A & N/A & 00001011 & N/A & $\mathrm{N} / \mathrm{A}$ & N/A & $\mathrm{N} / \mathrm{A}$ & 10001011 \\
\hline 72 & 01001011 & N/A & $\mathrm{N} / \mathrm{A}$ & 00101011 & 0101011 & N/A & N/A & $\mathrm{N} / \mathrm{A}$ & 10101011 \\
\hline 73 & 000000011 & $\mathrm{~N} / \mathrm{A}$ & N/A & 100000011 & 01000011 & N/A & $\mathrm{N} / \mathrm{A}$ & N/A & N/A \\
\hline 74 & 001000011 & N/A & N/A & 101000011 & 00010011 & N/A & N/A & N/A & N/A \\
\hline 75 & 100100011 & N/A & N/A & N/A & 00001011 & N/A & N/A & N/A & N/A \\
\hline 76 & 100010011 & 01010011 & N/A & N/A & 00101011 & 0101011 & N/A & N/A & N/A \\
\hline 77 & 101010011 & 01001011 & N/A & N/A & 100000011 & 01000011 & N/A & N/A & N/A \\
\hline 78 & N/A & 000000011 & $\mathrm{~N} / \mathrm{A}$ & N/A & 101000011 & 00010011 & $\mathrm{~N} / \mathrm{A}$ & N/A & $\mathrm{N} / \mathrm{A}$ \\
\hline 79 & $\mathrm{~N} / \mathrm{A}$ & 001000011 & N/A & N/A & N/A & 00001011 & N/A & N/A & N/A \\
\hline 80 & N/A & 100100011 & N/A & N/A & N/A & 00101011 & 0101011 & N/A & N/A \\
\hline 81 & N/A & 100010011 & 01010011 & N/A & N/A & 100000011 & 01000011 & N/A & N/A \\
\hline 82 & N/A & 101010011 & 01001011 & N/A & $\mathrm{N} / \mathrm{A}$ & 101000011 & 00010011 & $\mathrm{~N} / \mathrm{A}$ & N/A \\
\hline 83 & N/A & N/A & 000000011 & N/A & $\mathrm{N} / \mathrm{A}$ & N/A & 00001011 & N/A & N/A \\
\hline 84 & $\mathrm{~N} / \mathrm{A}$ & $\mathrm{N} / \mathrm{A}$ & 001000011 & $\mathrm{~N} / \mathrm{A}$ & N/A & N/A & 00101011 & 0101011 & N/A \\
\hline 85 & N/A & N/A & 100100011 & N/A & N/A & N/A & 100000011 & 01000011 & N/A \\
\hline 86 & 010000011 & N/A & 100010011 & 01010011 & $\mathrm{~N} / \mathrm{A}$ & N/A & 101000011 & 00010011 & $\mathrm{~N} / \mathrm{A}$ \\
\hline 87 & 000100011 & N/A & 101010011 & 01001011 & N/A & $\mathrm{N} / \mathrm{A}$ & N/A & 00001011 & N/A \\
\hline 88 & 000010011 & N/A & N/A & 000000011 & N/A & $\mathrm{N} / \mathrm{A}$ & N/A & 00101011 & 0101011 \\
\hline 89 & 001010011 & N/A & $\mathrm{N} / \mathrm{A}$ & 001000011 & N/A & N/A & N/A & 100000011 & 01000011 \\
\hline 90 & 100001011 & $\mathrm{~N} / \mathrm{A}$ & N/A & 100100011 & N/A & N/A & N/A & 101000011 & 00010011 \\
\hline 91 & 101001011 & N/A & N/A & 100010011 & 01010011 & N/A & N/A & N/A & 00001011 \\
\hline 92 & N/A & 010000011 & N/A & 101010011 & 01001011 & N/A & N/A & N/A & 00101011 \\
\hline 93 & N/A & 000100011 & N/A & N/A & 000000011 & N/A & N/A & N/A & 100000011 \\
\hline 94 & N/A & 000010011 & N/A & N/A & 001000011 & N/A & N/A & $\mathrm{N} / \mathrm{A}$ & 101000011 \\
\hline 95 & $\mathrm{~N} / \mathrm{A}$ & 001010011 & $\mathrm{~N} / \mathrm{A}$ & $\mathrm{N} / \mathrm{A}$ & 100100011 & N/A & $\mathrm{N} / \mathrm{A}$ & $\mathrm{N} / \mathrm{A}$ & N/A \\
\hline 96 & N/A & 100001011 & N/A & N/A & 100010011 & 01010011 & N/A & N/A & N/A \\
\hline 97 & N/A & 101001011 & N/A & N/A & 101010011 & 01001011 & N/A & N/A & N/A \\
\hline 98 & N/A & N/A & 010000011 & N/A & N/A & 000000011 & N/A & N/A & N/A \\
\hline 99 & N/A & $\mathrm{N} / \mathrm{A}$ & 000100011 & N/A & N/A & 001000011 & N/A & N/A & N/A \\
\hline 100 & 010100011 & $\mathrm{~N} / \mathrm{A}$ & 000010011 & N/A & N/A & 100100011 & N/A & $\mathrm{N} / \mathrm{A}$ & N/A \\
\hline
\end{tabular}

Proposition 3.11. For $a \leqslant-2$, there are seven straight lines $y+10 x=23+j$ for $j=1,2, \ldots, 7$ such that the seven points $(a, 24-10 a),(a, 25-10 a), \ldots,(a, 30-10 a)$ lie on these lines for $j=1,2, \ldots, 7$, respectively which give the respective GH codewords 0101000011, 0100100011, 0000010011, 0010010011, 1001010011, 1000001011, and 1010001011.

Proposition 3.12. For $a \leqslant-2$, there are seven straight lines $y+11 x=26+j$ for $j=1,2, \ldots, 7$ such that the seven points $(a, 27-11 a),(a, 28-11 a), \ldots,(a, 33-11 a)$ lie on these lines for $j=1,2, \ldots, 7$, respectively which give the respective GH codewords 0100010011, 0001010011, 0000001011, 0010001011, 1001001011, 1000101011, and 1010101011.

Proposition 3.13. For $a \leqslant-2$, there are seven straight lines $y+12 x=28+j$ for $j=1,2, \ldots, 7$ such that the seven points $(a, 29-12 a),(a, 30-12 a), \ldots,(a, 35-12 a)$ lie on these lines for $j=1,2, \ldots, 7$, respectively which give the respective GH codewords 0101010011, 0100001011, 0001001011, 0000101011, 0010101011, 10000000011, and 10100000011.

Proposition 3.14. For $a \leqslant-2$, there are seven straight lines $y+13 x=31+j$ for $j=1,2, \ldots, 7$ such that the seven points $(a, 32-13 a),(a, 33-13 a), \ldots,(a, 38-13 a)$ lie on these lines for $j=1,2, \ldots, 7$, respectively which give the respective GH codewords 0101001011, 0100101011, 00000000011, 00100000011, 10010000011, 10001000011, and 10101000011.

Thus we can determine the GH codeword of $(a, n), a \leqslant-2, n \leqslant 38-13 a$, if it exists from the above mentioned properties. 
Definition 3.15. The straight line $y+m x=c$ is called GH code straight line if all the integral points $(a, n)$ for $a \leqslant-2, n \geqslant 1$ on this straight line have GH codeword. Otherwise it is called Non-GH code straight line.

Without any loss of generality we assume that $m$ and $c$ both are non-negative integers.

Note 3.16. The point $(a, n)$ satisfying more than one GH code straight line, does not have unique GH codeword.

Theorem 3.17. The point $(a, n)$ gives the GH codeword $a_{1} a_{2} \ldots a_{l} 1$ if and only if the point $(a, n)$ satisfies the straight line $y+\left\{\sum_{k=1}^{l} a_{k} F(k-4)\right\} x=\sum_{k=1}^{l} a_{k} F(k-2)$ where $a_{k} \in\{0,1\}$ and the string $a_{1} a_{2} \ldots a_{l}$ does not contain any consecutive 1 's.

Proof. Let $(a, n)$ gives the codeword $a_{1} a_{2} \ldots a_{l} 1$. Then $n=\sum_{k=1}^{l} a_{k} V F_{a}(k)=\sum_{k=1}^{l} a_{k} F(k-2)$ $-a \sum_{k=1}^{l} a_{k} F(k-4)$, by Theorem 2.1. The point $\left(a, \sum_{k=1}^{l} a_{k} F(k-2)-a \sum_{k=1}^{l} a_{k} F(k-4)\right)$ lies on the straight line $y+\left\{\sum_{k=1}^{l} a_{k} F(k-4)\right\} x=\sum_{k=1}^{l} a_{k} F(k-2)$. Hence, the point $(a, n)$ lies on the straight line $y+\left\{\sum_{k=1}^{l} a_{k} F(k-4)\right\} x=\sum_{k=1}^{l} a_{k} F(k-2)$.

Conversely, let the point $(a, n)$ lies on the straight line $y+\left\{\sum_{k=1}^{l} a_{k} F(k-4)\right\} x=\sum_{k=1}^{l} a_{k} F(k-2)$. Therefore, for the point $(a, n)$, we have $n=\sum_{k=1}^{l} a_{k} F(k-2)-a \sum_{k=1}^{l} a_{k} F(k-4)=\sum_{k=1}^{l} a_{k} V F_{a}(k)$, by Theorem 2.1. Hence for $a \leqslant-2$, the GH codeword of $(a, n)$ is $a_{1} a_{2} \ldots a_{l} 1$ where $a_{k} \in\{0,1\}$ and the string $a_{1} a_{2} \ldots a_{l}$ does not contain any consecutive 1's.

Corollary 3.18. $\left(a, \mathrm{VF}_{\mathrm{a}}(\mathrm{k})\right)$ lies on the straight line $\mathrm{y}+\mathrm{F}(\mathrm{k}-4) \mathrm{x}=\mathrm{F}(\mathrm{k}-2)$ whose gradient is the Fibonacci number $-\mathrm{F}(\mathrm{k}-4)$.

\section{Determination of $\mathrm{GH}$ codeword for the point $(a, \mathfrak{n})$}

\subsection{Method 1}

In this section, we propose an algorithm which determines the GH codeword of a point $(a, n)$ where $a \leqslant-2$ and $n>38-13 a$, if it exists.

\section{Algorithm 4.1.}

Step 1. Take $r=1, m=n$.

Step 2. If $m=V F_{a}\left(i_{r}\right)$, then $G H$ codeword of $n$ always exists and is $\underbrace{00 \ldots 0}_{i_{r}-1 \text { times }} 11$ and then go to Step 12 .

Step 3. If $m-a=V F_{a}\left(i_{r}\right)$, then $G H$ codeword of $n$ always exists and is $\underbrace{00 \ldots 0}_{i_{r}-2 \text { times }} 11$ and then go to Step 12.

Step 4. If $m-a-1=V F_{a}\left(i_{r}\right)$, then GH codeword of $n$ always exists and is $101 \underbrace{00 \ldots 0}_{i_{r}-4 \text { times }} 11$ and then go to Step 12.

Step 5. Let $m_{r}=V F_{a}\left(i_{r}\right)$ be the greatest integer less than $m$.

Step 6. If $\left(m-m_{r}\right)>38-13 a$, then consider $m=m-m_{r}, r=r+1$ and go to Step 5 .

Step 7. If $m-m_{r}$ satisfies at least one straight line given in the Properties 3.1-3.14, then the GH codeword of $(a, n)$ exists and go to Step 8. Otherwise go to Step 9.

Step 8. The GH codeword corresponding to $(a, n)$ is the GH codeword of $\left(a, m-m_{r}\right)$ by deleting the last 1 then put 1 at the positions $i_{r}, i_{r-1}, \ldots, i_{1}$ and all other positions are zero and then put 1 at the last position and go to Step 12. 
Step 9. Let $m_{r+1}=V F_{a}\left(i_{r+1}\right)$, for some $k=i_{r+1}$, be the greatest number but not greater than $m_{r}$.

Step 10. Let $m-m_{r+1} \geqslant m_{r+1}$, then the GH codeword of $(a, n)$ does not exist and go to Step 12 .

Step 11. Otherwise $r=r+1$ and go to Step 5 .

Step 12. End.

Example 4.2. Determination of the GH codeword of $(-6,467)$ if it exists.

It is clear that $467 \notin V F_{-6}(k)$. By Step $5, m_{1}=293=V F_{-6}(12)$ is the greatest integer less than $467(=\mathrm{m})$. Now, $\mathrm{m}-\mathrm{m}_{1}=467-293=174>38-13(-6)$. By Step 6, $\mathrm{m}=174$ and by Step 5, $\mathrm{m}_{2}=112=\mathrm{VF}_{-6}(10)$ is the greatest integer less than 174 . Now, $\mathrm{m}-\mathrm{m}_{2}=174-112=62<38-13(-6)$ and $(-6,62)$ satisfies the straight line $y+7 x=15+5$ (Property 3.8).

Therefore the GH codeword of $(-6,62)$ is 100101011 . Hence by Step 8, the GH codeword of $(-6,467)$ is 1001010101011.

Example 4.3. Determination of the GH codeword of $(-5,683)$ if it exists.

It is obvious that $683 \notin V F_{-5}(k)$. By Step $5, m_{1}=678=V F_{-5}(14)$ is the greatest number less than $683(=\mathrm{m})$. We see that $\mathrm{m}-\mathrm{m}_{1}=683-678=5<38-13(-5)$ and $5<678$. But $(-5,5)$ does not satisfy any one of the straight lines given in the Properties 3.1-3.14. So, by Step 9, we take $m_{1}=419=V F_{-5}(13)$. Now, $m-m_{1}=683-419=264>38-13(-5)$. By Step $6, m=264$ and by Step $5, m_{2}=259=V F_{-5}(12)$ is the greatest number less than 264. Again, $m-m_{2}=264-259=5<38-13(-5)$ and $5<259$. So according to Step 10, we take $m_{2}=160=V F_{-5}(11)$. Now, $m-m_{2}=264-160=104>38-13(-5)$. By Step $6, m=104$ and by Step $5, m_{3}=99=V F_{-5}(10)$ is the greatest number less than 104 . Check that $\mathrm{m}-\mathrm{m}_{3}=104-99=5<38-13(-5)$ and $5<99$. So, by Step 9, we take $\mathrm{m}_{3}=61=V \mathrm{~F}_{-5}(9)$. Now, $\mathrm{m}-\mathrm{m}_{3}=104-61=43>38-13(-5)$ and $43<61$. But $(-5,43)$ does not satisfy any one of the straight lines given in the Properties 3.1-3.14. By Step 10, we take $m_{3}=38=V F_{-5}(8) . m-m_{3}=104-38=66<$ $38-13(-5)$ and $66>38$. By Step 10, the GH codeword of $(-5,683)$ does not exist.

\subsection{Determination of $c$ depending on $m(\geqslant 2)$ of the $G H$ code straight line $y+m x=c+j, 1 \leqslant j \leqslant 7$}

Let $(a, n)$ be any point on the GH code straight line $y+m x=c+1, m \geqslant 2$ and the GH codeword of $(a, n)$ be $a_{1} a_{2} \ldots a_{l} 1$ where $a_{k} \in\{0,1\}$ and the string $a_{1} a_{2} \ldots a_{l}$ does not contain any consecutive 1 's. From the Properties 3.1-3.14 and using Method 1, for the GH code straight line $y+m x=c+1$ we have always $a_{1}=0, a_{2}=1, a_{3}=0, a_{5}=0$. Now $n=\sum_{k=1}^{l} a_{k} V F_{a}(k)=\sum_{k=1}^{l} a_{k} F(k-2)-a \sum_{k=1}^{l} a_{k} F(k-4)$, by Theorem 2.1. Again by Theorem 3.17, $m=\sum_{k=1}^{l} a_{k} F(k-4)=a_{1} F(-3)+a_{2} F(-2)+a_{3} F(-1)+a_{4} F(0)+$ $\sum_{k=5}^{l} a_{k} F(k-4)=1+a_{4}+\sum_{k=5}^{l} a_{k} F(k-4)$, since $F(-3)=-1, F(-2)=1, F(-1)=0$ and $F(0)=1$. Therefore,

$$
m-1-a_{4}=\sum_{k=5}^{l} a_{k} F(k-4),
$$

where $a_{5} a_{6} \ldots a_{l}$ does not contain any consecutive $1^{\prime} s$. Thus $\sum_{k=5}^{l} a_{k} F(k-4)$ is the Zeckendorf's representation of $m-1-a_{4}$. Now, $a_{4}$ is either 0 or 1 . Hence, $\sum_{k=5}^{l} a_{k} F(k-4)$ is the Zeckendorf's representation of $m-2$ if and only if $a_{4}=1$ and $\sum_{k=5}^{l} a_{k} F(k-4)$ is the Zeckendorf's representation of $m-1$ if and only if $a_{4}=0$.

Again, $a_{5}=0$ always for the GH code straight line $y+m x=c+1$ and $m-2, m-1$ are consecutive positive integers.

There are three possible cases 0,$1 ; 1,0$; and 0,0 for the coefficients of $F(1)$ in the Zeckendorf's representation of $m-2$ and $m-1$, respectively. The case 1,1 is not possible, by Property 1.1.

Case 1: For any two consecutive positive integers $m-2$ and $m-1$, we consider the case that the coefficients of $F(1)$ in the Zeckendorf's representation of $m-2$ is 0 and $m-1$ is 1 . But in the equation (4.1) the 
coefficient of $F(1)$ for $m-1$ cannot be equal to 1 since $a_{5}=0$. Hence $\sum_{k=5}^{l} a_{k} F(k-4)$ is the Zeckendorf's representation of $m-2$.

Case 2: For any two consecutive positive integers $m-2$ and $m-1$, we consider the case that the coefficient of $F(1)$ in the Zeckendorf's representation of $m-2$ is 1 and $m-1$ is 0 . But in the equation (4.1) the coefficient of $F(1)$ for $m-2$ cannot be equal to 1 since $a_{5}=0$. Hence $\sum_{k=5}^{l} a_{k} F(k-4)$ is the Zeckendorf's representation of $m-1$.

Case 3: For any two consecutive positive integers $m-2$ and $m-1$, we consider the case that the coefficient of $F(1)$ in the Zeckendorf's representation of $m-2$ is 0 and $m-1$ is 0 .

Then the straight lines $y+4 x=7+1, y+9 x=20+1$ and $y+12 x=28+1$ for $m=4,9,12$ satisfy this case, i.e., the coefficient of $F(1)$ in the Zeckendorf's representation of $m-2$ is 0 as well as $m-1$ is 0 . For the straight line $y+4 x=7+1$, we have $a_{1}=0, a_{2}=1, a_{3}=0, a_{4}=1, a_{5}=0, a_{6}=1$ (by the Property 3.5) and $a_{5} F(1)+a_{6} F(2)$ is the Zeckendorf's representation of $4-2=2$. For the straight line $y+9 x=20+1$, we have $a_{1}=0, a_{2}=1, a_{3}=0, a_{4}=1, a_{5}=0, a_{6}=1, a_{7}=0, a_{8}=1$ (by the Property 3.10) and $a_{5} F(1)+a_{6} F(2)+a_{7} F(3)+a_{8} F(4)$ is the Zeckendorf's representation of $9-2=7$. For the straight line $y+12 x=28+1$, we have $a_{1}=0, a_{2}=1, a_{3}=0, a_{4}=1, a_{5}=0, a_{6}=1, a_{7}=0, a_{8}=0, a_{9}=1$ (by the Property 3.13) and $a_{5} F(1)+a_{6} F(2)+a_{7} F(3)+a_{8} F(4)+a_{9} F(5)$ is the Zeckendorf's representation of $12-2=10$. Hence in this case for $m \leqslant 13, \sum_{k=5}^{l} a_{k} F(k-4)$ is the Zeckendorf's representation of $m-2$.

Let $m \geqslant 14$. Then we have either $m-2=\sum_{k=5}^{l} a_{k} F(k-4)$ or $m-1=\sum_{k=5}^{l} a_{k} F(k-4)$. It is obvious that we have (i) $a_{5} F(1)+a_{6} F(2)=2$ or (ii) $a_{5} F(1)+a_{6} F(2)+a_{7} F(3)+a_{8} F(4)=7$ or (iii) $a_{5} F(1)+a_{6} F(2)+$ $a_{7} F(3)+a_{8} F(4)+a_{9} F(5)=10$ or (i) and (ii) or (i) and (iii). Thus we have always $a_{6}$, the coefficient of $F(2)$ is 1 . Hence by using Property $1.2, \sum_{k=5}^{l} a_{k} F(k-4)$ is the Zeckendorf's representation of $m-2$.

Therefore, we obtain the positive integer $m-1$ for the case 2 , otherwise we obtain the positive integer $m-2$.

Now from Theorem 3.17, we have

$$
c+1=1+a_{4} F(2)+\sum_{k=5}^{l} a_{k} F(k-2) .
$$

Therefore,

$$
c=2 a_{4}+\sum_{k=5}^{l} a_{k} F(k-2), \text { since } F(2)=2,
$$

where $\sum_{k=5}^{l} a_{k} F(k-4)$ is the Zeckendorf's representation of $m-2$ or $m-1$ according as $a_{4}=1$ or $a_{4}=0$. Hence, $c=\sum_{k=5}^{l} a_{k} F(k-2)+2$ if $\sum_{k=5}^{l} a_{k} F(k-4)$ is the Zeckendorf's representation of $m-2$ or $c=\sum_{k=5}^{l} a_{k} F(k-2)$ if $\sum_{k=5}^{l} a_{k} F(k-4)$ is the Zeckendorf's representation of $m-1$.

Thus we arrive at the following theorem.

Theorem 4.4. The GH codeword exists for $(a, n)$ if and only if $(a, n)$ satisfies at least one of the straight lines $\mathrm{y}+\mathrm{mx}=\mathrm{c}+\mathrm{j}$, where $\mathrm{m}, \mathrm{c}, \mathrm{j}$ are non-negative integers and

Case 1: $\mathrm{m}=0, \mathrm{c}=0$ and $\mathrm{j}=1,2,3,4$;

Case 2: $m=1, c=0$ and $j=1,2,3,4,5,6$;

Case 3: $m>1$. If the coefficient of $F(1)$ in the Zeckendorf's representation of $m-2$ is 0 , then $c=\sum_{k=1}^{l} a_{k} F(k+$ $2)+2$, where $\sum_{k=1}^{l} a_{k} F(k)$ is the Zeckendorf's representation of $m-2$, otherwise $c=\sum_{k=1}^{l} a_{k} F(k+2)$, where $\sum_{k=1}^{l} a_{k} F(k)$ is the Zeckendorf's representation of $m-1$ and for $m=F(4)+F(1), F(6)+F(1), F(6)+F(4)+F(1)$, $\mathrm{F}(7)+\mathrm{F}(4)+\mathrm{F}(1), \mathrm{F}(8)+\mathrm{F}(1), \mathrm{F}(8)+\mathrm{F}(4)+\mathrm{F}(1), \mathrm{F}(8)+\mathrm{F}(6)+\mathrm{F}(1), \mathrm{F}(8)+\mathrm{F}(6)+\mathrm{F}(4)+\mathrm{F}(1), \mathrm{F}(9)+\mathrm{F}(4)+$ $F(1), F(9)+F(6)+F(1), F(9)+F(6)+F(4)+F(1), F(9)+F(7)+F(4)+F(1), F(10)+F(1)+\cdots, j=1,2,3,4,5,6$, otherwise $\mathrm{j}=1,2,3,4,5,6,7$.

Proof.

Case 1: It follows from Property 3.1. 
Case 2: It follows from Property 3.2.

Case 3: It follows from 4.2 that if the coefficient of $F(1)$ in the Zeckendorf's representation of $m-2$ is 0 , then $c=\sum_{k=1}^{l} a_{k} F(k+2)+2$ where $\sum_{k=1}^{l} a_{k} F(k)$ is the Zeckendorf's representation of $m-2$ otherwise $c=\sum_{k=1}^{l} a_{k} F(k+2)$ where $\sum_{k=1}^{l} a_{k} F(k)$ is the Zeckendorf's representation of $m-1$.

Property 3.1 gives for $m=0, j=1,2,3,4$. Property 3.2 gives for $m=1=F(1), j=1,2,3,4,5,6$. Properties 3.3-3.14 give for $m=6=F(4)+F(1), j=1,2,3,4,5,6$ and for $m=2,3,4,5,7, \ldots, 13, j=$ $1,2,3,4,5,6,7$. Also from Theorem 3.17 we have $\left(a, V_{a}(k)\right)$ lies on the straight line $y+F(k-4) x=$ $\mathrm{F}(\mathrm{k}-2)$.

Hence by repeatedly applying Method 1 we have, for $m=F(4)+F(1), F(6)+F(1), F(6)+F(4)+F(1)$, $\mathrm{F}(7)+\mathrm{F}(4)+\mathrm{F}(1), \mathrm{F}(8)+\mathrm{F}(1), \mathrm{F}(8)+\mathrm{F}(4)+\mathrm{F}(1), \mathrm{F}(8)+\mathrm{F}(6)+\mathrm{F}(1), \mathrm{F}(8)+\mathrm{F}(6)+\mathrm{F}(4)+\mathrm{F}(1), \mathrm{F}(9)+\mathrm{F}(4)+$ $F(1), F(9)+F(6)+F(1), F(9)+F(6)+F(4)+F(1), F(9)+F(7)+F(4)+F(1), F(10)+F(1)+\cdots, j=1,2,3,4,5,6$, otherwise $j=1,2,3,4,5,6,7$.

The GH codewords for $0 \leqslant m \leqslant 14$ are given in Table 5 .

The GH code straight lines for $m=0,1,2,3,4,5,6,7,8$ are given in Figure 1 .

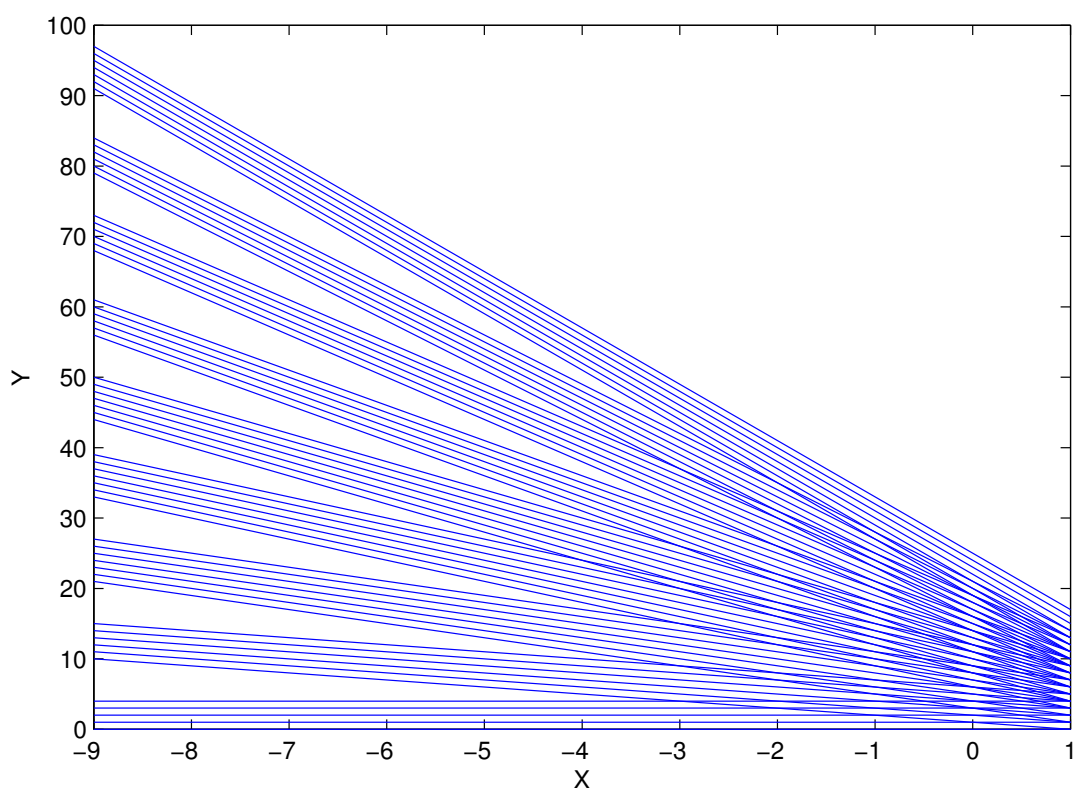

Figure 1: GH code straight lines.

Corollary 4.5. The GH codeword of $(a, n)$ is the codeword corresponding to the $G H$ code straight line $y+m x=$ $c+j$ satisfied by $(a, n)$.

Corollary 4.6. The GH codeword of $(a, n)$ is not unique if $(a, n)$ satisfies more than one GH code straight line.

Theorem 4.7. GH codeword of $(a, n)$ is not unique for $-4 \leqslant a \leqslant-2$.

Proof. GH codeword of $(a, n)$ is not unique if $(a, n)$ lies on at least two GH code straight lines. If $(a, n)$ lies on the GH code straight lines $y+m_{1} x=c_{1}+j_{1}$ and $y+m_{2} x=c_{2}+j_{2}$, where $m_{1}<m_{2}$, then $a=\frac{\left(c_{2}+j_{2}\right)-\left(c_{1}+j_{1}\right)}{m_{2}-m_{1}}$. Therefore,

$$
c_{2}+j_{2}<c_{1}+j_{1},
$$

since $a \leqslant-2$ and $m_{1}<m_{2}$. 
Table 5: The GH codewords for $0 \leqslant m \leqslant 14$.

\begin{tabular}{|c|c|c|c|c|c|c|c|}
\hline $\mathrm{m}$ & $\mathrm{c}(\mathrm{m})$ & j & GH code & $\mathrm{m}$ & $\mathrm{c}(\mathrm{m})$ & $\mathrm{j}$ & GH code \\
\hline \multirow[t]{4}{*}{0} & 0 & 1 & 0011 & 8 & 18 & 1 & 010001011 \\
\hline & & 2 & 10011 & & & 2 & 000101011 \\
\hline & & 3 & 100011 & & & 3 & 0000000011 \\
\hline & & 4 & 101011 & & & 4 & 0010000011 \\
\hline \multirow[t]{6}{*}{1} & 0 & 1 & 011 & & & 5 & 1001000011 \\
\hline & & 2 & 00011 & & & 6 & 1000100011 \\
\hline & & 3 & 000011 & & & 7 & 1010100011 \\
\hline & & 4 & 001011 & 9 & 20 & 1 & 010101011 \\
\hline & & 5 & 1000011 & & & 2 & 010000011 \\
\hline & & 6 & 1010011 & & & 3 & 0001000011 \\
\hline \multirow[t]{7}{*}{2} & 2 & 1 & 01011 & & & 4 & 0000100011 \\
\hline & & 2 & 010011 & & & 5 & 0010100011 \\
\hline & & 3 & 0000011 & & & 6 & 1000010011 \\
\hline & & 4 & 0010011 & & & 7 & 1010010011 \\
\hline & & 5 & 1001011 & 10 & 23 & 1 & 0101000011 \\
\hline & & 6 & 10000011 & & & 2 & 0100100011 \\
\hline & & 7 & 10100011 & & & 3 & 0000010011 \\
\hline \multirow[t]{7}{*}{3} & 5 & 1 & 0100011 & & & 4 & 0010010011 \\
\hline & & 2 & 0001011 & & & 5 & 1001010011 \\
\hline & & 3 & 00000011 & & & 6 & 1000001011 \\
\hline & & 4 & 00100011 & & & 7 & 1010001011 \\
\hline & & 5 & 10010011 & 11 & 26 & 1 & 0100010011 \\
\hline & & 6 & 10001011 & & & 2 & 0001010011 \\
\hline & & 7 & 10101011 & & & 3 & 0000001011 \\
\hline \multirow[t]{7}{*}{4} & 7 & 1 & 0101011 & & & 4 & 0010001011 \\
\hline & & 2 & 01000011 & & & 5 & 1001001011 \\
\hline & & 3 & 00010011 & & & 6 & 1000101011 \\
\hline & & 4 & 00001011 & & & 7 & 1010101011 \\
\hline & & 5 & 00101011 & 12 & 28 & 1 & 0101010011 \\
\hline & & 6 & 100000011 & & & 2 & 0100001011 \\
\hline & & 7 & 101000011 & & & 3 & 0001001011 \\
\hline \multirow[t]{7}{*}{5} & 10 & 1 & 01010011 & & & 4 & 0000101011 \\
\hline & & 2 & 01001011 & & & 5 & 0010101011 \\
\hline & & 3 & 000000011 & & & 6 & 10000000011 \\
\hline & & 4 & 00100011 & & & 7 & 10100000011 \\
\hline & & 5 & 100100011 & 13 & 31 & 1 & 0101001011 \\
\hline & & 6 & 100010011 & & & 2 & 0100101011 \\
\hline & & 7 & 101010011 & & & 3 & 00000000011 \\
\hline \multirow[t]{6}{*}{6} & 13 & 1 & 010000011 & & & 4 & 00100000011 \\
\hline & & 2 & 000100011 & & & 5 & 10010000011 \\
\hline & & 3 & 000010011 & & & 6 & 10001000011 \\
\hline & & 4 & 001010011 & & & 7 & 10101000011 \\
\hline & & 5 & 100001011 & 14 & 34 & 1 & 01000000011 \\
\hline & & 6 & 101001011 & & & 2 & 00011000011 \\
\hline \multirow[t]{7}{*}{7} & 15 & 1 & 010100011 & & & 3 & 00001000011 \\
\hline & & 2 & 010010011 & & & 4 & 00101000011 \\
\hline & & 3 & 000001011 & & & 5 & 100001000011 \\
\hline & & 4 & 001001011 & & & 6 & 10100100011 \\
\hline & & 5 & 100101011 & & & & \\
\hline & & 6 & 1000000011 & & & & \\
\hline & & 7 & 1010000011 & & & & \\
\hline
\end{tabular}

We have from the equations of the GH code straight lines, for $a \leqslant-2,(4.2)$ is possible only when $m_{1}$ and $m_{2}$ are consecutive, i.e., $m_{2}-m_{1}=1$, otherwise $a>-2$. Therefore, $a=\left(c_{2}+j_{2}\right)-\left(c_{1}+j_{1}\right) \leqslant-2$. Then $j_{2}-j_{1} \leqslant-4$ or -5 , since $c_{2}-c_{1}=2$ or 3 , by Theorem 4.4. So $j_{2}-j_{1}=-4,-5$ or -6 , since $1 \leqslant j_{1}, j_{2} \leqslant 7$. Hence $a=-2,-3,-4$. 
Thus the GH codeword of $(a, n)$ is not unique for $-4 \leqslant a \leqslant-2$.

Theorem 4.8. $(a, n), a \leqslant-2$ satisfies maximum two GH code straight lines.

Proof. We know that, the gradients of two intersecting GH code straight lines for $-4 \leqslant a \leqslant-2$ must be consecutive. Hence, $(a, n), a \leqslant-2$ satisfies maximum two GH code straight lines.

Corollary 4.9. The maximum GH codewords of $(a, n)$ are two if the codeword of $(a, n)$ exists.

Note 4.10. We have one alternative codewords of the green colored codewords of $(a, n)$ 's in Tables 1 and 2.

For an example, $(-3,9)$ has two codewords: 1010011 and 01011.

Corollary 4.11. The GH codeword of $(a, n)$ if exists, is unique for $a \leqslant-5$.

\subsection{Method 2}

\section{Algorithm 4.12.}

Step 1. Check whether $(a, n)$ satisfies at least one of the GH code straight line $y+m x=c+j$ or not. If it satisfies, the GH codeword of $(a, n)$ exists otherwise not.

Step 2. Write down the GH code straight line.

Step 3. Find the point on that straight line when $a=-5$ (we can choose any value of $a \leqslant-5$ ). Let the point be $\left(-5, n_{1}\right)$.

Step 4. Determine the GH codeword of $\left(-5, n_{1}\right)$ by using Algorithm 4.1. The GH codeword of $\left(-5, n_{1}\right)$ is the GH codeword of $(a, n)$.

Example 4.13. Determine the GH codeword of the point $(-600,4560)$, if it exits.

Let $(-600,4560)$ satisfies the straight line $y+m x=c+j$. Then the maximum value of $m$ is $\left[\frac{4560}{600}\right]=7$. Therefore $c=F(6)+2=13+2=15$, since $m-2=5=F(4)$. From the equation we have, $j=345>7$. Therefore $(-600,4560)$ does not satisfy any GH code straight line and hence GH codeword of $(-600,4560)$ does not exist.

Example 4.14. Determine the GH codeword of the point $(-1745,15728)$, if it exits.

Let $(-1745,15728)$ satisfies the straight line $y+m x=c+j$. Then the maximum value of $m$ is $\left[\frac{15728}{1745}\right]=$ 9. Therefore $c=F(4)+F(6)+2=5+13+2=20$ since $m-2=7=F(2)+F(4)$. So $j=3<7$. Therefore $(-1745,15728)$ satisfies the GH code straight line $y+9 x=20+3$ and hence GH codeword of $(-1745,15728)$ exists. Now the GH code straight line $y+9 x=20+3$ gives $y=68$ when $x=-5$. Thus the GH codeword of $(-1745,15728)$ is 0001000011 since Table 2 gives the GH codeword of $(-5,68)$ which is 0001000011.

\section{Conclusion}

For the sequence $\mathrm{VF}_{-5}(\mathrm{k})$, Thomas showed that it is not possible to write the Zeckendorf's representation for integers 5 and 12. Thereafter, Basu and Prasad improved the availability of GH code upto positive integer 100 for $-20 \leqslant a \leqslant-2$. But there was no general method to determine the GH codeword for any integer $a \leqslant-2$ and any positive integer $n$. In this paper, we have introduced the idea of GH code straight line. Then we proposed a couple of algorithms to determine the existence of GH codewords. If $\mathrm{GH}$ codeword exists, then it is easy to find the codeword for a given parameter $(a, n)$, using algorithms mentioned in this paper. 


\section{Acknowledgment}

We thank the editor and the anonymous referees for their valuable and insightful comments and suggestions. We are also thankful to Dr Satya Bagchi for his suggestions to improve the quality of the manuscript. The first named author Joydeb Pal is thankful to DST-INSPIRE for financial support to pursue his research work.

\section{References}

[1] M. Basu, B. Prasad, Long range variations on the Fibonacci universal code, J. Number Theory, 130 (2010), 1925-1931. 1

[2] D. E. Daykin, Representation of natural numbers as sums of generalized Fibonacci numbers, J. Lond. Math. Soc., 35 (1960), 143-160. 2

[3] P. Elias, Universal codeword sets and representations of the integers, IEEE Trans. Information Theory, 21 (1975), 194-203. 1

[4] S. Kak, A ristotle and Gautama on logic and physics, arxiv, 2005 (2005), 16 pages. 2

[5] S. Kak, Greek and Indian cosmology: Review of early history, arxiv, 2005 (2005), 38 pages. 2

[6] D. Knuth, Negafibonacci Numbers and the Hyperbolic Plane, Paper presented at the annual meeting of the Mathematical Association of America, San Jose, (2008). 2

[7] I. G. Pearce, Indian mathematics: Redressing the balance, University of St. Andrews, U. K., (2002). 2

[8] J. H. Thomas, Variation on the Fibonacci universal code, arXiv, 2007 (2007), 4 pages. 1

[9] E. Zeckendorf, Representation des nombres naturels par une somme des nombres de Fibonacci ou de nombres de Lucas (French), Bull. Soc. Roy. Sci. Liege, 41 (1972), 179-182. 1 